\title{
Homocysteine and carotid intima-media thickness in ischemic stroke patients are not correlated
}

\author{
George Ntaios' \\ Christos Savopoulos' \\ Apostolos Hatzitolios' \\ Ippoliti Ekonomou ${ }^{2}$ \\ Evangelos Destanis ${ }^{2}$ \\ loannis Chryssogonidis ${ }^{2}$ \\ Anastasia Chatzinikolaou ${ }^{3}$ \\ Ifigenia Pidonia ${ }^{3}$ \\ Dimitrios Karamitsos' \\ 'First Propedeutic Department of \\ Internal Medicine; ${ }^{2}$ Department \\ of Radiology; ${ }^{3}$ Department of \\ Biochemistry, AHEPA Hospital, \\ Aristotle University of Thessaloniki, \\ Thessaloniki, Greece
}

\begin{abstract}
Introduction: Hyperhomocysteinemia has been linked to cardiovascular morbidity and mortality by numerous authors. Whether this association is causal or not remains uncertain. The aim of the study was to investigate the association of hyperhomocysteinemia with the degree of carotid atherosclerosis in stroke patients.

Methods: We studied 97 Greek patients in our stroke unit who were hospitalized as a result of ischemic stroke between March 2006 and May 2007. The patients were divided into two groups: the first (52 patients) included stroke patients with serum levels of homocysteine below $15 \mu \mathrm{mol} / \mathrm{L}$, but in the second group (45 patients) serum homocysteine exceeded this value. We measured carotid intima-media thickness (cIMT) in all patients and correlated it with serum homocysteine.
\end{abstract}

Results: The mean homocysteine concentration was $11.5 \mu \mathrm{mol} / \mathrm{L}$ in the first group and $21.5 \mu \mathrm{mol} / \mathrm{L}$ in the second group. Carotid IMT was $1.012 \mathrm{~mm}$ in the first group, and $1.015 \mathrm{~mm}$ in the second group, an insignificant difference. On the contrary, serum folate concentration was $21.3 \mathrm{nmol} / \mathrm{L}$ in the first group compared with $16.7 \mathrm{nmol} / \mathrm{L}$ in the second group $(\mathrm{p}<0.001)$. VitB12 was $401 \mathrm{pmol} / \mathrm{L}$ in the first group and $340 \mathrm{pmol} / \mathrm{L}$ in the second group, a statistically significant difference $(\mathrm{p}<0.001)$.

Conclusions: Serum levels of homocysteine were not correlated with cIMT in ischemic stroke patients. Both folate and vitB12 were decreased in hyperhomocysteinemic ischemic stroke patients.

Keywords: homocysteine, carotid intima-media thickness, ischemic stroke

\section{Introduction}

Hyperhomocysteinemia has been linked to cardiovascular morbidity and mortality by numerous authors (Matetzky et al 2003). Whether this association is causal or not remains uncertain. Homocysteine is a sulfur-containing amino acid which can either be remethylated to methionine, or transulphurated to cysteine. In the first case, folic acid and cobalamin (vitB12) are used as coenzymes, whereas in the latter pathway pyridoxine (vitB6) is utilized (Finkelstein 2000).

Homocysteine levels below $15 \mu \mathrm{mol} / \mathrm{L}$ are considered normal. Hyperhomocysteinemia has been classified as follows (Kang et al 1992): 15-30 $\mu \mathrm{mol} / \mathrm{L}$, moderate; 30-100 $\mu \mathrm{mol} / \mathrm{L}$, intermediate; higher than $100 \mu \mathrm{mol} / \mathrm{L}$, severe.

This study aimed to investigate the association between hyperhomocysteinemia and the degree of carotid atherosclerosis in stroke patients.

\section{Methods}

We studied 97 Greek patients (51 males, 46 females) in our stroke unit who were hospitalized as a result of ischemic stroke from March 2006 to May 2007. The mean age of the patients was 72.8 years. The patients were divided in two groups. The first included 52 stroke patients with serum levels of homocysteine below $15 \mu \mathrm{mol} / \mathrm{L}$. 
In the second group, which included 45 patients, serum homocysteine exceeded this value. In all cases, the diagnosis of ischemic stroke was confirmed by computer tomography of the brain. Major exclusion criteria included renal failure, thyroid disease, pregnancy, hepatic failure, tumors, and drugs such as antiepileptics, fibrates, hormone replacement therapy, folate, vitB12, and vitB6 which are known to influence homocysteine levels (Dierkes and Westphal 2005).

Measurements of carotid intima-media thickness (cIMT) were performed in the far wall of both carotids at three points: a) the carotid bifurcation, b) the common carotid arteries, $1 \mathrm{~cm}$ proximal to the dilatation of the carotid bulb, and c) the internal carotid arteries, $1 \mathrm{~cm}$ away from the bifurcation.

The study was approved by the Ethics Committee of our hospital.

\section{Results}

The mean serum homocysteine concentration and the mean cIMT of the patients in our study were $17.4 \mu \mathrm{mol} / \mathrm{L}$ and $1.013 \mathrm{~mm}$, respectively. Table 1 sets out the main clinical and biochemical parameters for the two groups. The mean homocysteine concentration was $11.5 \mu \mathrm{mol} / \mathrm{L}$ in the first group and $21.5 \mu \mathrm{mol} / \mathrm{L}$ in the second group. The difference in cIMT between the two groups was not significant. More specifically, cIMT in the first group was $1.012 \mathrm{~mm}$, and $1.015 \mathrm{~mm}$ in the second group.

In contrast, the levels of serum folate concentration in the two groups differed significantly ( $\mathrm{p}<0.001)$. As expected, the level was higher in the first group $(21.3 \mathrm{nmol} / \mathrm{L})$, but significantly lower in the second group $(16.7 \mathrm{nmol} / \mathrm{L})$.

Table I Mean values of main clinical and biochemical parameters in the two groups

\begin{tabular}{lll}
\hline & Group I $(\mathbf{n}=\mathbf{5 2})$ & Group II $(\mathbf{n}=\mathbf{4 7})$ \\
\hline Age $($ years $)$ & 72.6 & 72.9 \\
BMI $\left(\mathrm{kg} / \mathrm{m}^{2}\right)$ & 28.4 & 28.1 \\
SBP $(\mathrm{mmH})$ & $16 \mathrm{I})$ & 166 \\
DBP $(\mathrm{mmHg})$ & 89 & 92 \\
Homocysteine $(\mu \mathrm{mol} / \mathrm{l})$ & 11.5 & $21.5^{*}$ \\
Folate $(\mathrm{nmol} / \mathrm{l})$ & 21.3 & $16.7^{*}$ \\
VitBI2 $(\mathrm{pmol} / \mathrm{l})$ & $40 \mathrm{I}$ & $340^{*}$ \\
Total Cholesterol $(\mathrm{mg} / \mathrm{dl})$ & 157 & 160 \\
LDL $(\mathrm{mg} / \mathrm{dl})$ & 114 & 116 \\
HDL $(\mathrm{mg} / \mathrm{dl})$ & 50 & 47 \\
Triglycerides $(\mathrm{mg} / \mathrm{dl})$ & 152 & 148 \\
Serum Creatinine $(\mathrm{mg} / \mathrm{dl})$ & $0.9 \mathrm{I}$ & 0.94 \\
cIMT $(\mathrm{mm})$ & $\mathrm{I} .012$ & 1.015 \\
\hline
\end{tabular}

Abbreviations: BMI, body mass index; SBP, systolic blood pressure; DBP, diastolic blood pressure; cIMT, carotid intima-media thickness.

Notes: *statistically significant difference $(\mathrm{p}<0.00 \mathrm{I})$.
Moreover, the levels of vitB12 were also statistically different between the two groups $(p<0.001)$. The mean value of vitB12 was $401 \mathrm{pmol} / \mathrm{L}$ in the first group and $340 \mathrm{pmol} / \mathrm{L}$ in the second group.

\section{Discussion}

Our results indicate that serum levels of homocysteine do not correlate with cIMT in ischemic stroke patients. Therefore compared with those with low levels of homocysteine, the hyperhomocysteinemic stroke patients of our study presented with similar degree of atherosclerosis, as indicated by cIMT, which is considered to be a reliable marker of the atherosclerotic process (Aminbakhsh and Mancini 1999).

A second conclusion was the reciprocal relationship between homocysteine and $\mathrm{B}$ vitamins, namely folate and vitamin B12. Both folate and vitamin B12 levels were raised in the first group, but were significantly lower in the second group. This result was not surprising, because both folate and vitamin B12 play an important role in the metabolism of homocysteine to methionine and cysteine, respectively (Finkelstein 2000). Our results confirm previous studies that reached the same conclusion (Boushey et al 1994; Vermeulen et al 2000; Till et al 2005; Marcucci et al 2005).

There is still no consensus on whether homocysteine is a causative risk factor for cardiovascular events like stroke and coronary disease. Numerous epidemiological studies have correlated hyperhomocysteinemia to cardiovascular morbidity and mortality. A meta-analysis indicated that when homocysteine increases by $5 \mu \mathrm{mol} / \mathrm{L}$, the relative cardiovascular risk increases by a factor of 1.6 and 1.8 in men and women, respectively (Brattstrom et al 1990; Boushey et al 1994). The same study estimated that $10 \%$ of the total cardiovascular risk could be attributed to hyperhomocysteinemia. In the Atherosclerosis Risk in Communities (ARIC) study, hyperhomocysteinemia was correlated with increased coronary atherosclerosis (Chambless et al 2002). The Perth Carotid Ultrasound Disease Assessment Study (CUDAS) associated homocysteine levels with carotid intima media thickness in 1111 asymptomatic patients (McQuillan et al 1999). After adjustment for age, sex, and other conventional risk factors, subjects in the highest versus the lowest quartile of homocysteine had an odds ratio of 2.60 for increased IMT and 1.76 for carotid artery plaque. The authors of this study proposed that mild hyperhomocysteinemia is an independent risk factor for increased carotid artery wall thickness and plaque formation in the general population. However, other recent studies failed to confirm this result. More specifically, Durga and colleagues (2004) found no 
correlation between homocysteine and IMT in the general population in Netherlands. Voutilainen and colleagues (1998) found a positive association between homocysteine and cIMT only in men, but not in women.

Folic acid and vitamin B12 are important factors in the homocysteine metabolism. Boushey and colleagues (1994) showed that administration of folate, vitamin B6, or vitamin B12 decreased homocysteine levels by $42 \%, 5 \%$, and $15 \%$, respectively within 6 weeks. In 1998, the United States Food and Drug Administration (FDA) required all cereal-grain products to be fortified with folic acid (Grosse et al 2005). The intention was that the typical daily ingestion of folate would increase by approximately $100 \mu \mathrm{g}$.

To summarize, our study failed to confirm the association of hyperhomocysteinemia with cIMT in ischemic stroke patients. Large, prospective, multicentre studies are needed to more thoroughly investigate the role of homocysteine in the atherosclerotic process.

\section{References}

Aminbakhsh A, Mancini GB. 1999. Carotid intima-media thickness measurements: what defines an abnormality? A systematic review. Clin Invest Med, 22:149-57.

Boushey C, Beresford SAA, Omenn G, et al. 1994. A quantitative assessment of homocysteine as a risk factor for vascular disease. Probable benefits from increasing folate intakes. JAMA, 274:1049-57.

Brattstrom L, Israelsson B, Norrving B, et al. 1990. Impaired homocysteine metabolism in early-onset cerebral and peripheral occlusive arterial disease. Effects of pyridoxine and folic acid treatment. Atherosclerosis, 81:51-60.
Chambless LE, Folsom AR, Davis V, et al. 2002. Risk factors for progression of common carotid atherosclerosis: the Atherosclerosis Risk In Communities Study, 1987-1998. Am J Epidemiol, 155:38-47

Dierkes J, Westphal S. 2005. Effects of drugs on Homocysteine concentrations. Semin Vasc Med, 5:124-39

Durga J, Verhoef P, Bots ML, et al. 2004. Homocysteine and carotid intimamedia thickness: A critical appraisal of the evidence. Atherosclerosis, 176:1-19

Finkelstein JD. 2000. Pathways and regulation of homocysteine metabolism in mammals. Semin Thromb Hemost, 26:219-25.

Grosse SD, Waitzman NJ, Romano PS, et al. 2005. Reevaluating the benefits of folic acid fortification in the United States: Economic analysis, regulation, and public health. Am J Public Health, 95:1917-22.

Kang SS, Wong PWK, Malinow MR. 1992. Hyperhomocysteinemia as a risk factor for occlusive vascular disease. Ann Rev Nutr, 12:279-98.

Marcucci R, Zanazzi M, Bertoni E, et al. Homocysteine-lowering therapy and carotid intima-media thickness in renal transplant recipients. Transplantation Proceedings 2005 July-August; 37:2491-2.

Matetzky S, Freimark D, Ben-Ami S, et al. 2003. Association of elevated homocysteine levels with a higher risk of recurrent coronary events and mortality in patients with acute myocardial infarction. Arch Intern Med, 163:1933-7.

McQuillan BM, Beilby JP, Nidorf M, et al. 1999. Hyperhomocysteinemia but not the C677T mutation of methylenetetrahydrofolate reductase is an independent risk determinant of carotid wall thickening. The Perth Carotid Ultrasound Disease Assessment Study (CUDAS). Circulation, 99:2383-8.

Till U, R $\varphi$ hl P, Jentsch A, et al. 2005. Decrease of carotid intima-media thickness in patients at risk to cerebral ischemia after supplementation with folic acid, vitamins B6 and B12. Atherosclerosis, 181:131-5.

Vermeulen EG, Stehouwer CD, Twisk JW, et al. 2000. Effect of homocysteinelowering treatment with folic acid plus vitamin B6 on progression of subclinical atherosclerosis: a randomised, placebo-controlled trial. Lancet, 355:517-22

Voutilainen S, Alfthan G, Nyyssonen K, et al. 1998. Association between elevated plasma total Homocysteine and increased common carotid artery wall thickness. Ann Med, 30:300-5. 
